\title{
Silicon-organic hybrid (SOH) integration and photonic multi-chip systems: Technologies for high-speed optical interconnects
}

\author{
C. Koos ${ }^{1,2, *}$, W. Freude ${ }^{1}$, J. Leuthold ${ }^{3}$, L. R. Dalton ${ }^{4}$, S. Wolf ${ }^{1}$, H. Zwickel ${ }^{1,2}$, T. Hoose ${ }^{2}$, M. R. Billah', \\ M. Lauermann ${ }^{1}$, C. Weimann ${ }^{1}$, W. Hartmann ${ }^{1,2}$, A. Melikyan ${ }^{2}$, N. Lindenmannn', S. Koeber ${ }^{1,2}$, \\ R. Palmer ${ }^{1}$, D. Korn ${ }^{1}$, L. Alloatti ${ }^{1}$, A.-L. Giesecke ${ }^{6}$, T. Wahlbrink ${ }^{6}$ \\ ${ }^{1}$ Institute of Photonics and Quantum Electronics (IPQ), Karlsruhe Institute of Technology (KIT), 76131 Karlsruhe, Germany \\ ${ }^{2}$ Institute of Microstructure Technology (IMT), Karlsruhe Institute of Technology (KIT), 76131 Karlsruhe, Germany \\ ${ }^{3}$ Now with: Institute of Electromagnetic Fields (IEF), Swiss Federal Institute of Technology (ETH), Zurich, Switzerland \\ ${ }^{4}$ University of Washington, Department of Chemistry, Seattle, WA 98195-1700, United States \\ ${ }^{6}$ AMO GmbH, 52074 Aachen, Germany \\ *email: christian.koos@kit.edu
}

\begin{abstract}
Limitations of silicon photonics can be overcome by hybrid integration of silicon photonic or plasmonic circuits with organic materials or by photonic multi-chip systems. We give an overview on our recent progress regarding both siliconorganic hybrid (SOH) integration and multi-chip integration enabled by photonic wire bonding.
\end{abstract}

Keywords - Silicon photonics, silicon-organic hybrid (SOH) integration, plasmonic-organic hybrid (SOH) integration, multichip integration, photonic wire bonding, optical interconnects

\section{SUMMARY}

Silicon photonics shows tremendous potential for largescale photonic-electronic integration by fabless fabrication of photonic and electronic circuits [1]. Silicon as an optical material, however, falls short of properties that are indispensable for high-performance devices: The indirect bandgap of crystalline silicon inhibits efficient light emission, and the inversion symmetry of the silicon crystal lattice prevents second-order optical nonlinearities, thereby making electro-optic modulators challenging. Our research focusses on hybrid integration concepts, which combine silicon photonic circuits with other material systems that provide complementary optical properties. In this paper, we give an overview on our recent progress in the fields of silicon-based hybrid integration and of photonic multi-chip integration.

To enable efficient electro-optic modulators on the silicon photonic platform, we exploit the concept of silicon-organic hybrid (SOH) integration that combines nanophotonic silicon waveguides with organic cladding materials [2] - [15]. This approach [2] leads to highly efficient devices, featuring energy consumptions of only a few fJ per bit [3], [4]. The response of the electro-optic materials is ultra-fast and enables small-signal modulation at $100 \mathrm{GHz}$ [5], generation of $100 \mathrm{Gbit} / \mathrm{s}$ on-ofkeying (OOK) signals [6], and symbol rates of 64 GBd for multi-level signaling [7]. Moreover, we demonstrated generation of advanced modulation formats such as 16QAM using in-phase-quadrature (IQ-)modulators with record-low energy consumption and symbol rates (bit rates) of up to $40 \mathrm{GBd}$ (160 Gbit/s) [8], [9]. We further show that the extraordinarily low operating voltage of $\mathrm{SOH}$ modulators allows operation of these devices directly from standard output ports of field-programmable gate arrays (FPGA), without the need for external amplifiers and digital-to-analog converters. Such schemes can be used even if higher-order modulation formats such as 16QAM are to be generated [10]. Moreover, we use SOH devices for generating broadband frequency combs, which are well suited as optical multi-wavelength sources for terabit/s transmission [11]. On the long run, organic electro-optic materials might be replaced by second-order nonlinear metamaterials that exploit highly stable inorganic ABC-type nanolaminates fabricated by atomiclayer deposition (ALD) [12].

The $\mathrm{SOH}$ approach is a versatile concept that goes far beyond electro-optic modulators. In particular, we have also shown that compact and power-efficient $\mathrm{SOH}$ phase shifters can realized by using liquid crystals (LC) as cladding materials [13]. Moreover, optically pumped lasers have been demonstrated on the silicon photonic platform by using $\mathrm{SOH}$ waveguides that are based on dyedoped cladding materials [14]. These devices can only be operated in pulsed mode and lend themselves as sources in optical biosensors. We have also shown that the concept of $\mathrm{SOH}$ integration can be transferred to plasmonic waveguide structures, leading to plasmonic-organic hybrid $(\mathrm{POH})$ devices [15]. We have demonstrated $\mathrm{POH}$ Mach-Zehnder modulators that feature a flat frequency response up to at least $65 \mathrm{GHz}$. Such devices and will potentially open the route for modulation at $\mathrm{THz}$ frequencies [15].

(c) 2016 IEEE. Personal use of this material is permitted. Permission from IEEE must be obtained for all other uses, in any current or future media, including reprinting/republishing this material for advertising or promotional purposes, creating new collective works, for resale or redistribution to servers or lists, or reuse of any copyrighted component of this work in other works. See http://www.ieee.org/publications_standards/publications/rights/index.html for more information. 
This article has been accepted for publication, but has not been fully edited. Content may change prior to final publication. Citation information: DOI https://doi.org/10.1109/OIC.2016.7483033

The concepts of $\mathrm{SOH}$ and $\mathrm{POH}$ integration are complemented by photonic multi-chip integration that exploits photonic wire bonding [16] for assembling photonic systems from discrete dies fabricated on different material platforms [17], [18]. We have introduced photonic wire bonding and demonstrated its viability for chip-chip [16], [18] and fiber-chip [17] interfaces. The concept enables, e.g., hybrid multi-chip integration of silicon photonic circuitry along with InP-based light sources [18]. We believe that hybrid integration and photonic multichip systems are key concepts for highly scalable terabit/s photonic transceiver systems.

\section{ACKNOWLEDGEMENTS}

This work was supported by the European Research Council (ERC Starting Grant 'EnTeraPIC', number 280145), by the Alfried Krupp von Bohlen und Halbach Foundation, by the EU projects PhoxTroT and BigPipes, by the BMBF project PHOIBOS, by the Collaborative Research Centre "WavePhenomena" (CRC 1173) of Deutsche Forschungsgemeinschaft (DFG), by the Helmholtz International Research School for Teratronics (HIRST), by the Karlsruhe School of Optics and Photonics (KSOP), and by the Karlsruhe Nano-Micro Facility (KNMF).

\section{REFERENCES}

[1] Sun, C.; Wade, M. T.; Lee, Y.; Orcutt, J. S.; Alloatti, L.; Georgas, M. S.; Waterman, A. S.; Shainline, M. S.; Avizienis, R. R.; Lin, S.; Moss, B. R.; Kumar, R.; Pavanello, F.; Atabaki, A. H.; Cook, H. M.; Ou, A. J.; Leu, J. C.; Chen, Y.-H.; Asanović, K.; Ram, R. J.; Popović, M. A.; Stojanović, V. M.. 'Single-chip microprocessor that communicates directly using light" Nature 528, 534-538 (2015)

[2] Koos, C.; Leuthold, J.; Freude, W.; Kohl, M.; Dalton, L. R.; Bogaerts, W.; Giesecke, A.-L-; Lauermann, M.; Melikyan, A.; Koeber, S.; Wolf, S.; Weimann, C.; Muehlbrandt, S.; Koehnle, K.; Pfeifle, J.; Hartmann, W.; Kutuvantavida, Y.; Ummethala, S.; Palmer, R.; Korn, D.; Alloatti, L.; Schindler, P. C.; Elder, D. L.; Wahlbrink, T.; Bolten, J.: 'Silicon-Organic Hybrid (SOH) and Plasmonic-Organic Hybrid (POH) Integration', J. Lightw. Technol., DOI: 10.1109/JLT.2015.2499763 (2016)

[3] Palmer, R.; Koeber, S.; Elder, D. L.; Woessner, M.; Heni, W.; Korn, D.; Lauermann, D.; Bogaerts, W.; Dalton, L. R.; Freude, W.; Leuthold, J.; Koos, C.: 'High-Speed, Low Drive-Voltage Silicon-Organic Hybrid Modulator based on a BinaryChromophore Electro-Optic Material', J. Lightw .Technol. 32, 2726-2734 (2014)

[4] Koeber, S.; Palmer, R.; Lauermann, M.; Heni, W.; Elder, D.; Korn, D.; Woessner, M; Alloatti, L.; Koenig, S.; Schindler, P.C.; Yu, H.; Bogaerts, W.; Dalton, L.-R.; Freude, W.; Leuthold, J.; Koos, C.: 'Femtojoule electro-optic modulation using a silicon-organic hybrid device'; Light: Science \& Applications 4, e255 (2015)

[5] Alloatti, L.; Palmer, R.; Diebold, S.; Pahl, K. P.; Chen, B.; Dinu, R.; Fournier, M.; Fedeli, J.-M.; Zwick, T.; Freude, W.; Koos, C.; Leuthold, J.: ' $100 \mathrm{GHz}$ silicon-organic hybrid modulator,' Light: Science \& Applications 3, e173 (2014)

[6] Hartmann, W.; Lauermann, M.; Wolf, S.; Zwickel, H.; Kutuvantavida, Y.; Luo, J.; Jen, A. K.-Y.; Freude, W.; Koos, C.:
'100 Gbit/s OOK using a silicon-organic hybrid (SOH) modulator,' 41th European Conf. Opt. Commun. (ECOC'15), Valencia, Spain, Sept. 27 - Oct. 5, 2015. Paper PDP1.4

[7] Lauermann, M.; Wolf, S.; Palmer, R.; Bielik, A.; Altenhain, L.; Lutz, J.; Schmid, R.; Wahlbrink, T.; Bolten, J.; Giesecke, A. L.; Freude, W.; Koos, C.: '64 GBd operation of a silicon-organic hybrid modulator at elevated temperature,' Optical Fiber Communication Conference (OFC'15), Los Angeles (CA), USA, 22.-26.03.2015. Paper Tu2A.5

[8] Lauermann, M.; Palmer, R.; Koeber, S.; Schindler, P. C.; Korn, D.; Wahlbrink, T.; Bolten, J.; Waldow, M.; Elder, D. L.; Dalton, L. R.; Leuthold, J.; Freude, W.; Koos, C.: 'Low-power siliconorganic hybrid (SOH) modulators for advanced modulation formats,' Opt. Express 22, 29927-29936 (2014)

[9] Lauermann, M.; Wolf, S.; Schindler, P.C.; Palmer, R.; Koeber, S.; Korn, D.; Alloatt, L.; Wahlbrink, T.; Bolten, J.; Waldow, M.; Koenigsmann, M.; Kohler, M.; Malsam, D.; Elder, D.L.; Johnston, P.V.; Phillips-Sylvain, N.; Sullivan, P.A.; Dalton, L. R.; Leuthold, J.; Freude, W.; Koos, C.: '40 GBd 16QAM Signaling at 160 Gbit/s in a Silicon-Organic Hybrid (SOH) Modulator,', J. Lightw. Technol. 33, 1210-1216 (2015)

[10] Wolf, S.; Lauermann, M.; Schindler, P.C.; Ronniger, G.; Geistert, K.; Palmer, R.; Koeber, S.; Bogaerts, W.; Leuthold, J.; Freude, W.; Koos, C.: "DAC-less Amplifier-less Generation and Transmission of QAM Signals Using Sub-Volt Silicon-organic Hybrid Modulators," J. Lightw. Technol. 33, 1425-1432 (2015)

[11] Weimann, C.; Schindler, P.; Palmer, R.; Wolf, S.; Bekele, D.; Korn, D.; Pfeifle, J.; Koeber, S.; Schmogrow, R.; Alloatti, L.; Elder, D.; Yu, H.; Bogaerts, W.; Dalton, L.; Freude, W.; Leuthold, J.; and Koos, C.: 'Silicon-organic hybrid (SOH) frequency comb sources for terabit/s data transmission,' Opt. Express 22, 3629-3637 (2014).

[12] Alloatti, L.; Kieninger, C.; Froelich, A.; Lauermann, M.; Frenzel, T.; Köhnle, K.; Freude, W.; Leuthold, J.; Wegener, M.; Koos, C.: 'Second-order nonlinear optical metamaterials: ABCtype nanolaminates,' Appl. Phys. Lett. 107, 121903-1-4 (2015)

[13] Pfeifle, J.; Alloatti, L.; Freude, W.; Leuthold, J. and Koos, C.: 'Silicon-organic hybrid phase shifter based on a slot waveguide with a liquid-crystal cladding,' Opt. Express 20 (2012), 1535915376

[14] Korn, D.; Lauermann, M.; Koeber, S.; Appel, P.; Alloatti, L.; Palmer, R.; Dumon, P.; Freude, W.; Leuthold, J.; Koos, C.: "Lasing in Silicon-Organic Hybrid (SOH) Waveguides"; Nature Communications (2016)

[15] Melikyan, A.; Koehnle, K.; Lauermann, M.; Palmer, R.; Koeber, S.; Muehlbrandt, S.; Schindler, P. C.; Elder, D. L.; Wolf, S.; Heni, W.; Haffner, C.; Fedoryshyn, Y.; Hillerkuss, D.; Sommer, M.; Dalton, L. R.; Thourhout, D. V.; Freude, W.; Kohl, M.; Leuthold, J.; Koos, C.: 'Plasmonic-organic hybrid (POH) modulators for OOK and BPSK signaling at 40 Gbit/s,' Opt. Express 23, 9938-9946 (2015)

[16] Lindenmann, N.; Balthasar, G.; Hillerkuss, D.; Schmogrow, R.; Jordan, M.; Leuthold, J.; Freude, W.; Koos, C.: 'Photonic wire bonding: a novel concept for chip-scale interconnects,' Opt. Express 20, 17667-17677 (2012)

[17] Lindenmann, N.; Dottermusch, S.; Goedecke, M.-L.; Hoose, T.; Billah, M.-R.; Onanuga, T.-P; Hofmann, A.; Freude, W.; Koos, C.: 'Connecting silicon photonic circuits to multicore fibers by photonic wire bonding;' J. Lightw. Technol. 33, 755-760 (2015)

[18] Billah, M. R.; Hoose, T.; Onanuga, T.; Lindenmann, N.; Dietrich, P.; Wingert, T.; Goedecke, M. L.; Hoffmann, A.; Troppenz, U.; Sigmund, A.; Möhrle, M.; Freude, W.; Koos, C.: 'Multi-chip integration of lasers and silicon photonics by photonic wire bonding,' Conf. on Lasers and Electro-Optics (CLEO'15), San Jose (CA), USA, May 10-15, 2015. Paper STu2F.2

(C) 2016 IEEE. Personal use of this material is permitted. Permission from IEEE must be obtained for all other uses, in any current or future media, including reprinting/republishing this material for advertising or promotional purposes, creating new collective works, for resale or redistribution to servers or lists, or reuse of any copyrighted component of this work in other works.

See http://www.ieee.org/publications_standards/publications/rights/index.html for more information. 